\title{
A simple and scalable method to generate spheroids from human mesenchymal stem cells for use in tissue engineering
}

\author{
Ngoc Bich $\mathrm{Vu}^{1,2, *(\mathbb{C}, \text { Minh Thi-Nguyet Nguyen }}{ }^{1,2}$
}

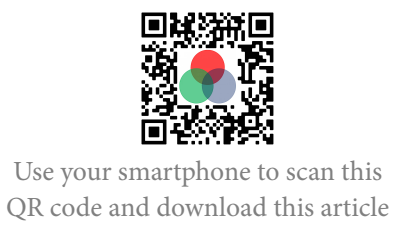

${ }^{1}$ Stem Cell Institute, University of Science, Ho Chi Minh City, Viet Nam

${ }^{2}$ Vietnam National University Ho Chi Minh City, Viet Nam

\section{Correspondence}

Ngoc Bich Vu, Stem Cell Institute, University of Science, Ho Chi Minh City, Viet Nam

Vietnam National University Ho Chi Minh City, Viet Nam

Email: ngocvu@sci.edu.vn

\section{History}

- Received: 2020-10-01

- Accepted: 2020-12-01

- Published: 2020-12-29

DOI : 10.15419/bmrat.v7i12.652

\section{Check for updates}

\section{Copyright}

( $)$ Biomedpress. This is an openaccess article distributed under the terms of the Creative Commons Attribution 4.0 International license.

\begin{abstract}
Introduction: Tissue engineering is a field suited for applying stem cells, besides stem cell transplantation. In the current tissue engineering approaches, stem cells are typically seeded onto a suitable scaffold and induced into specific tissues under particular conditions. However, this strategy has faced some limitations, namely that stem cell proliferation on the scaffolds' surface has been inefficient to fill the porous scaffolds to produce solid tissues. Some limitations have been improved by using stem cell spheroids on the scaffold in place of single stem cells. This study aimed to evaluate a simple and feasible method to produce spheroids of mesenchymal stem cells (MSCs) from adipose and umbilical cord tissues for use in tissue engineering. Methods: MSCs from human adipose tissue (adipose-derived stem cells, i.e., ADSCs) and human umbilical cord tissues (umbilical cord-derived mesenchymal stem cells, i.e., UCMSCs) were isolated according to previously published protocols. To produce spheroids, ADSCs and UCMSCs were cultured in non-adherent Vbottom 96-well plate. Three cell densities were evaluated: 250 cells/well, 500 cells/well, and 1,000 cells/well. The generated spheroids were evaluated based on spheroid diameter, necrotic core formation (using propidium iodide (PI) and Hoechst 33342 staining), and spheroid structure (by Hematoxylin \& Eosin staining). Results: The results showed that at a density of 250 cells/well, spheroids were formed without necrotic cores from both ADSCs and UCMSCs. However, at a higher density, all spheroids had a necrotic core as part of the three zones (proliferating, quiescent, and necrotic zones). Conclusion: Spheroids from ADSCs and UCMSCs can be easily produced by culturing 250 cells/well in a non-adherent V-bottom 96-well plate. This process can be scaled up by using the liquid handling robot system to load cells into the plates.

Key words: Adipose-derived stem cells, Microtissue, Spheroids, Three - dimensional cell culture, Umbilical cord-derived mesenchymal stem cells
\end{abstract}

\section{INTRODUCTION}

Cell therapy and tissue engineering are considered significant advances for regenerative medicine, drug discovery, and cell-based biosensors ${ }^{1}$. To date, most of the recent studies are often based on twodimensional (2D) culture. However, experimental studies in 2D do not mimic the natural physiological characteristics of cells and tissues in vivo and are limited in simulating cells' interaction with their environment ${ }^{2}$. The technological research advances in regenerative medicine have allowed the creation of threedimensional (3D) in vitro model, which better reflect the in vivo cell signaling interactions than the $2 \mathrm{D}$ monolayer cell culture model ${ }^{1,3}$, as well as facilitating an improved microenvironment for cell growth ${ }^{4}$. Nowadays, 3D cell culture has become a new trend in basic and applied research, with the number of studies in this area, increasing each day significantly.

Many studies on spheroid formation, evaluation, and application have been carried out on cancer cell lines, such as MCF7 ${ }^{5,6}$, HCT116, HepG2, MDA-MB-231 ${ }^{7}$,
BT-474, T-47D, and MDA-MB-361 cells ${ }^{6}$. However, studies on spheroid-derived mesenchymal stem cells (MSCs) have been limited. MSCs have great potential for use in regenerative medicine and tissue engineering. Indeed, MSCs derived from human adipose tissues (adipose-derived stem cells, i.e., ADSCs) and umbilical cord tissues (umbilical cord-derived mesenchymal stem cells, i.e., UCMSCs) have shown enormous potential in various applications ${ }^{8}$. As MSCs, they possess similar essential characteristics; however, ADSCs and UCMSCs have many distinct features that affect their applications. Due to differences in the acquisition, isolation, and potential activities of ADSCs versus UCMSCs, when evaluating the efficiency of microtissue models, such as the 3D model, it is necessary to conduct a direct comparison of the two MSC sources to confirm which source is more efficient ${ }^{9-12}$.

Traditionally, engineered tissues like cartilage and bone have been produced by seeding MSCs onto a scaffold under inducible conditions. However, a new, 
simple, and highly efficient method has yielded engineered tissues from stem cell spheroids. Winter et al. (2003) reported on cartilage production via differentiated human stem cell spheroids ${ }^{13}$. Moreover, Yoon et al. (2012) suggested that in spheroids, ADSCs could be triggered to undergo chondrogenesis via hypoxia-related cascades and enhanced cell-cell interactions ${ }^{11}$. Hyaline-like cartilage tissue also could be created from ADSCs cultured on poly(L-glutamic acid)/chitosan scaffold ${ }^{14}$ or in the porous scaffold ${ }^{15}$. Furthermore, Stuart et al. (2017) reported that engineered cartilage could be produced at a low-cost using progenitor cell spheroids ${ }^{16}$. Recently, our group has successfully created cartilage tissues from scaffoldfree ADSC-derived spheroids ${ }^{17}$.

Moreover, the engineered bone tissues can be produced in similar ways to engineered cartilage tissues using stem cell spheroids. In 2013, Shen et al. (2013) reported the successful production of spheroids derived from ADSCs and the ability to induce osteogenic differentiation in these spheroids ${ }^{18}$. Laschke et al. (2014) also tried to use ADSC-derived spheroids and porous scaffolds to produce bone tissues ${ }^{19}$. Interestingly, ectopic bones could be formed in animals when engineered bones based on MSC-derived spheroids were combined with calcium phosphate microparticles and platelet-rich plasma hydrogel $^{20}$.

In general, there are two main kinds of MSC-derived spheroids (scaffold-based spheroids and scaffold-free spheroids), which, to date, can be produced by various means. Methods to produce MSC-based spheroids include spontaneous formation in a 48 -well plate ${ }^{13}$, in combination with a poly(L-glutamic acid)/chitosan scaffold ${ }^{14}$, use of a spinner flask ${ }^{11}$, use of porous scaffold $^{15}$, hanging droplet method ${ }^{18}$, pellet culture ${ }^{21}$, and centrifugation ${ }^{22}$. These methods, however, are complicated for scale-up production to obtain adequate numbers of spheroids needed for further experimentation and applications. Therefore, this study aimed to evaluate a simple method of generating MSC spheroids based on culturing the cells in nonadherent V-bottom 96-well plates and tested the scaleup process via liquid handling robots to produce a high number of spheroids.

\section{MATERIALS - METHODS}

Human adipose-derived and human umbilical cord-derived mesenchymal stem cells

The human ADSCs and human UCMSCs used in this study were previously established by our laboratory ${ }^{23-26}$. The cells were thawed and characterized before use in experiments. ADSCs and UCMSCs were cultured T75 flasks containing MSCCult
I medium (Regenmedlab, Ho Chi Minh City, VN). The two cell lines were allowed to proliferate in an incubator $\left(37^{\circ} \mathrm{C}, 5 \% \mathrm{CO}_{2}\right)$, replenishing fresh MSCCult I medium every 2-3 days.

To verify their MSC phenotype, the ADSCs and UCMSCs were characterized by flow cytometry (FCM) using standard cell surface markers. Briefly, cells were detached by treatment with Deattachment Reagent (Regenmedlab, Ho Chi Minh City, VN) for 3 minutes, then resuspended in Washing Buffer (Regenmedlab, Ho Chi Minh City, VN), and centrifuged at $1,500 \mathrm{rpm}$ for 5 minutes. Cell pellets were resuspended in FCM washing/staining buffer (Regenmedlab, Ho Chi Minh City, VN) and stained individually with the amount in $5 \mu \mathrm{L}$ of the appropriate antibodies room temperature for 30 minutes. The antibodies included: CD90-PE, CD105-FITC, CD14-FITC, or CD73-PerCP-CP 5.5 (all purchased from Santa Cruz Biotechnology, Dallas, TX), or with CD34-FITC, CD45-APC, CD44-APC, and HLA-DRFITC (all purchased from BD Biosciences, San Jose, CA). Then, $200 \mu \mathrm{l}$ of BD FACSFlow ${ }^{\mathrm{TM}}$ Sheath Fluid (BD Biosciences) was added to each tube. The stained cells were run on a BD FACSCalibur machine (BD Biosciences) and the data analyzed using CellQuest Pro software (BD Biosciences), at a minimum of 1,000 events. Isotype controls were used for all tests.

To evaluate the in vitro differentiation potential of ADSCs and UCMSCs, the cells were cultured in 3 types of inducing media: adipogenic-, osteogenic- or chondrogenic-differentiation media. For adipogenic differentiation, the two cell lines were treated with an adipogenic-induction medium (StemPro adipogenesis Differentiation Media) (Gibco/ ThermoFisher Scientific, Waltham, MA). The medium was changed every 6-7 days. After 12 to 14 days, cell differentiation was assessed by staining with Oil Red O (Sigma Aldrich, St. Louis, MO). For osteogenic differentiation, the cells were cultured in an osteogenicinduction medium (StemPro osteogenesis Differentiation Media) (Gibco/ ThermoFisher Scientific). The medium was replenished every seven days. On day 30, the cell differentiation was assessed by staining with Alizarin Red S (Sigma-Aldrich). Lastly, to facilitate induction into chondroblasts, the cells were treated in a chondrogenic-induction medium (StemPro ${ }^{\circ}$ Chondrogenesis Differentiation Media) (Gibco/ ThermoFisher Scientific). The medium was replenished every seven days, and at day 30, cell differentiation was assessed by staining with Alcian Blue (Sigma-Aldrich). 


\section{Spheroid formation and characterization}

Non-adherent V-bottom 96-well plates were used in our spheroid formation method. Cells were seeded at three different densities ( 250 cells/well, 500 cells/well, or 1,000 cells/well). The experiment was repeated at least three times for each cell density group. After seeding the cells into 96 -well plates $(100 \mu \mathrm{l} /$ well in each well of a 96-well plate), the spheroid diameter was measured and recorded using the inverted microscope (Olympus, Japan). From here, we determined the optimal density of cells for spheroid seeding for the subsequent experiments.

\section{Assessment of death and survival rates of the ADSCs and UCMSCs}

After spheroid formation, the viability of ADSCs and UCMSCs was assessed by propidium iodide (PI) and Hoechst 33342 double staining; both reagents were purchased from Sigma-Aldrich. Cell samples were fixed in paraformaldehyde (PFA, 4\%) (SigmaAldrich) and incubated for 30 minutes at $4^{\circ} \mathrm{C}$. Then, the samples were rinsed in saline sodium citrate buffer (SSC, 2X) containing $0.03 \mathrm{M}$ sodium citrate and 0.3 $\mathrm{M}$ sodium chloride (Sigma-Aldrich) to completely remove the PFA. A total of $50 \mu \mathrm{l}$ RNase solution (SigmaAldrich) was added in, and the samples were incubated for 20 minutes at $37^{\circ} \mathrm{C}$. After rinsing the samples with 2 X SSC, $50 \mu \mathrm{l}$ of $1 \mu \mathrm{g} / \mathrm{mL}$ Hoechst 33342 solution was added into each sample well and incubated at $37^{\circ} \mathrm{C}, 5 \% \mathrm{CO}_{2}$. After 30 minutes, the samples were rinsed in 2X SSC following PI cell staining (with 50 $\mu \mathrm{l}$ of the final one $\mathrm{ng} / \mu \mathrm{l}$ PI solution) for 30 minutes at $37^{\circ} \mathrm{C}, 5 \% \mathrm{CO}_{2}$. Finally, the samples were washed in 2X SSC several times. The spheroids were imaged using a ZEISS LSM 880 Confocal Laser Scanning Microscope (Carl-Zeiss, Germany).

\section{Statistical analysis}

The data are presented graphically as mean $\pm \mathrm{SD}$. GraphPad Prism software (version 7.0 for Windows; GraphPad Software, Inc., La Jolla, CA, USA) was used for statistical analysis of the results. All results were evaluated for statistical significance at a significance level of 0.05 (95\% confidence level).

\section{RESULTS}

\section{Characterization of human ADSCs and UCMSCs}

The results showed that after thawing, both UCMSCs (Figure 1A) and ADSCs (Figure 1E) adhered to and spread with fibroblast-like morphology along the plastic surface of the flasks. When cultured in various differentiation media, both ADSCs and UCMSCs showed the ability to differentiate in vitro along the mesenchymal trilineage, including adipocytes, osteocytes, and chondrocytes. In the osteogenesisinducing conditions of ADSCs (Figure 1F) and UCMSCs (Figure 1B), there was an accumulation of calcium mineralization in the extracellular matrix (ECM), as was observed by the formation of red complexes after staining with Alizarin Red dye. This showed that ADSCs and UCMSCs could differentiate into osteocytes. During the adipogenesisinducing process, from day 8 to 14 , both ADSCs (Figure 1G) and UCMSCs (Figure 1C) formed lipid droplets inside the cytoplasm, which stained positive with Oil Red O dye. This indicated that ADSCs and UCMSCs could differentiate into adipocytes. In the chondrogenesis-differentiation medium, the induced cells expressed protein aggrecans that bind with $\mathrm{Al}$ cian Blue dye to form blue complexes (Figure 1D, H). This showed that ADSCs and UCMSCs could differentiate into chondrocytes.

FCM analysis results showed that both ADSCs (Figure 2A) and UCMSCs (Figure 2B) were negative for CD14, CD34, CD45, and HLA-DR surface expression, with the percentages falling below $2 \%$. However, they were positive for CD90, CD44, CD73, and CD105, with the percentages being more significant than 95\%. The above results confirmed that ADSCs and UCMSCs, indeed, exhibited the surface marker phenotype of MSCs.

\section{The spheroid-forming efficiency of ADSCs and UCMSCs}

Two hours after seeding into non-adhesion 96-well plates, ADSCs were aggregated into loose clusters and allowed to settle down to the plate's bottom. Twentyfour hours later, the ADSC clusters could be formed from 3 cell densities (250, 500, and 1,000 cells/well). At this time, the cell clusters could easily be broken by mechanical impact. On day 3, the cell clusters continued to shrink in size, and the cells in the 250 cells/well and 500 cells/well density groups exhibited a spherical morphology. There was no sight of the individual cells in both of these density groups. The spherical morphology was maintained throughout the culture process. However, for the density group of 1,000 cells/well, the cells did not form a clear spherical shape.

Observing the growth size of the ADSC spheroids over a 10-day period (Figure 3S, Table 1), it is evident that in all three cell density groups, the spheroid size 

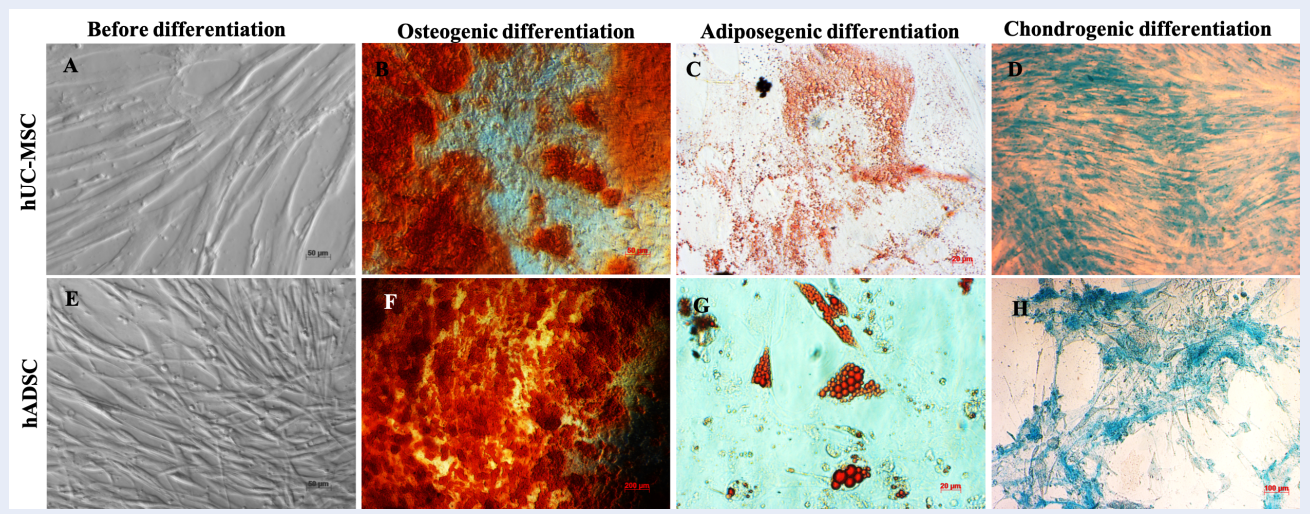

Figure 1: Morphology and in vitro differentiation potential of ADSCs and UCMSCs. (A, E) ADSCs and UCMSCS displayed fibroblast-like cell morphology. The cells could be differentiated into osteoblasts, which stained positive with Alizarin Red staining (B, F), into adipocytes, which stained positive with Oil Red O staining (C, G), and into chondroblasts, which were positive by Alcian Blue staining (D, H).

tended to decrease from the day 1 to day two greatly. There were significant changes $(\mathrm{p}<0.0001)$ compared to the remaining days (days $3,5,7$, and 10 ). At the density of 250 cells/well, the average size of spheroids decreased from $703.883 \pm 70.975 \mu \mathrm{m}$ (at day 1) to $423.847 \pm 38.544 \mu \mathrm{m}$ (at day 10 ). There was no statistically significant reduction in the mean spheroid size from days 3, 5, and 7 (560.501 $\pm 48.422 \mu \mathrm{m}, 518.52$ $\pm 47.81 \mu \mathrm{m}$, and $512.16 \pm 39.45 \mu \mathrm{m}$, respectively) till day 10 ( $\mathrm{p}>0.05)$. At the 500 cells/well density, the spheroid size was reduced from $866.310 \pm 62.546$ $\mu \mathrm{m}$ (day 1 ) to $625.228 \pm 40.207 \mu \mathrm{m}$ (day 10). At the 1,000 cells/well density, the average spheroid size did not follow any patterns or rules. The spheroid size was $1585.381 \pm 124.054 \mu \mathrm{m}$ (at day 1 ), $960.75 \pm 81.14 \mu \mathrm{m}$ (at day 3), $1218.63 \pm 75.18 \mu \mathrm{m}$ (at day 5), $825.2 \pm$ $75.82 \mu \mathrm{m}$ (at day 7), and $624.1 \pm 32.77 \mu \mathrm{m}$ (day 10). Thus, these results showed that ADSC spheroids could be formed from day three by the cell seeding densities of 250 and 500 cells/well. From day 3 to day 7 of culture, the spheroid sizes ranged from 423.85 to 560.5 $\mu \mathrm{m}$ when the cell seeding density was 250 cells/well and ranged from 625.23 to $769.09 \mu \mathrm{m}$ when the seeding density was 500 cells/well (Figure 3S).

Meanwhile, single UCMSCs at a density of 250, 500, or 1,000 cells/well, seeded in 96-well plate (Figure 2AC), showed aggregation at day one and formation of spheroid-like structures (Figure 4D-F). However, some single cells or small clusters present at the outer edges of these spheroid-like structures. From day 3, the outer edges of the spheroid appeared smoother (Figure 4G-I). There were no single cells or small clusters adhered to the spheroids. The structures were maintained until day 10 for all three densities (Figure 4 J-R).

From observing the UCMSC spheroids' growth over the 10-day period (Figure 4S; Table 2), significant differences in size could be seen for all three seeding density groups. For both the 500 cells/well and 1,000 cells/well densities, the spheroid size tended to decrease over time. At the 500 cells/well density, the average size of spheroids decreased over time- from $429.24 \pm 41.71 \mu \mathrm{m}$ (day 1 ) to $332 \pm 30.16 \mu \mathrm{m}$ (day 10). For the 1,000 cells/well density, the average size of spheroids decreased from $547.66 \pm 50.72 \mu \mathrm{m}$ (day 1) to $423.88 \pm 40.33 \mu \mathrm{m}$ (day 7). After that time, there was no statistically significant change in spheroid size. Particularly, at the 250 cells/well density, the average size of spheroids showed no significant change in the culture over time, with the size range being maintained from $311.3 \pm 28.39 \mu \mathrm{m}$ (day 1) to $323.2 \pm 24.5$ $\mu \mathrm{m}$ (day 7).

Therefore, these results show that human UCMSC spheroids could be formed from day one from all three seeding densities (250, 500, and 1,000 cells/well). The spheroid sizes were about $300 \mu \mathrm{m}$ (for 250 cells/well density); the size reached 332 to 429.24 $\mu \mathrm{m}$ (for 500 cells/well density group), and 423.88 to $547.66 \mu \mathrm{m}$ (for 1,000 cells/well density group) during the 10-day period.

\section{MSC spheroid structures}

On day 3, the cell distribution of ADSC spheroids for all three cell density groups $(250,500$, and 1,000 cells/well) was not uniform (Figure 5A, C, and E, respectively). The cell density was still sparse, especially at the core of the spheroids. Notably, in the 

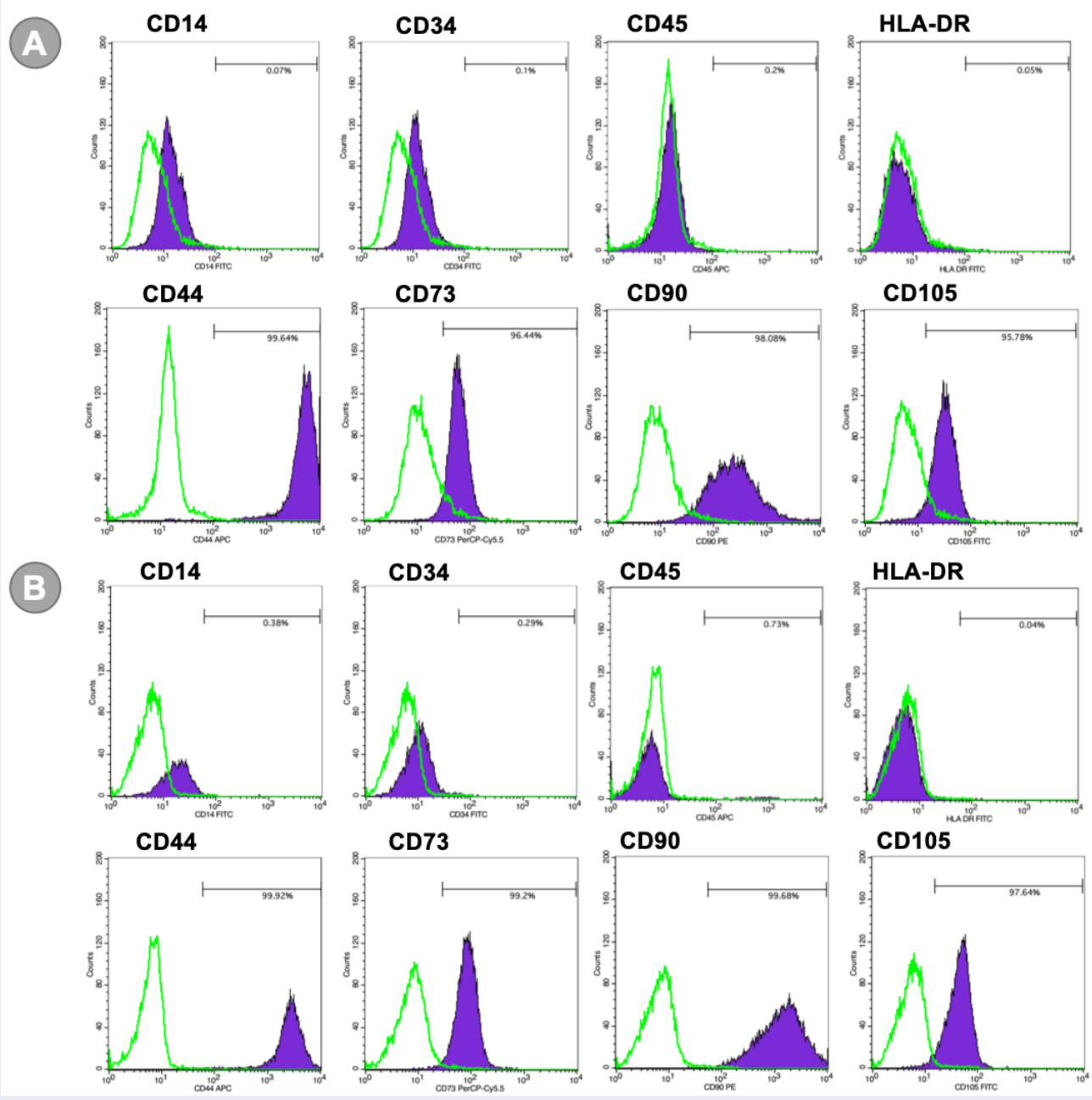

Figure 2: Cell surface marker expression. Flow cytometry analyses for MSC markers showed that both ADSCs (A) and UCMSCs (B) were negative for CD14, CD34, CD45, and HLA-DR surface expression but were positive for CD44, CD73, CD90, and CD105 surface expression.

Table 1: ADSC spheroids formed at different cell densities

\begin{tabular}{ccccccc}
\hline $\begin{array}{c}\text { Cell seed } \\
\text { density }\end{array}$ & $\begin{array}{r}\text { Spheroid size at 250 cells } \\
(\mu \mathbf{m})\end{array}$ & \multicolumn{2}{c}{$\begin{array}{r}\text { Spheroid size at 500 cells } \\
(\mu \mathbf{m})\end{array}$} & \multicolumn{2}{c}{$\begin{array}{r}\text { Spheroid size at 1,000 cells } \\
(\mu \mathbf{m})\end{array}$} \\
\hline Time (Day) & Mean & SD & Mean & SD & Mean & SD \\
1 & 703.88 & 70.97 & 866.31 & 62.55 & 1585.38 & 124.05 \\
3 & 560.50 & 48.42 & 726.66 & 71.87 & 960.70 & 81.14 \\
5 & 518.52 & 47.81 & 769.09 & 76.73 & 1218.63 & 75.18 \\
7 & 512.16 & 39.45 & 668.77 & 64.63 & 825.20 & 75.82 \\
10 & 423.85 & 38.54 & 625.23 & 40.21 & 624.10 & 32.77 \\
\hline
\end{tabular}




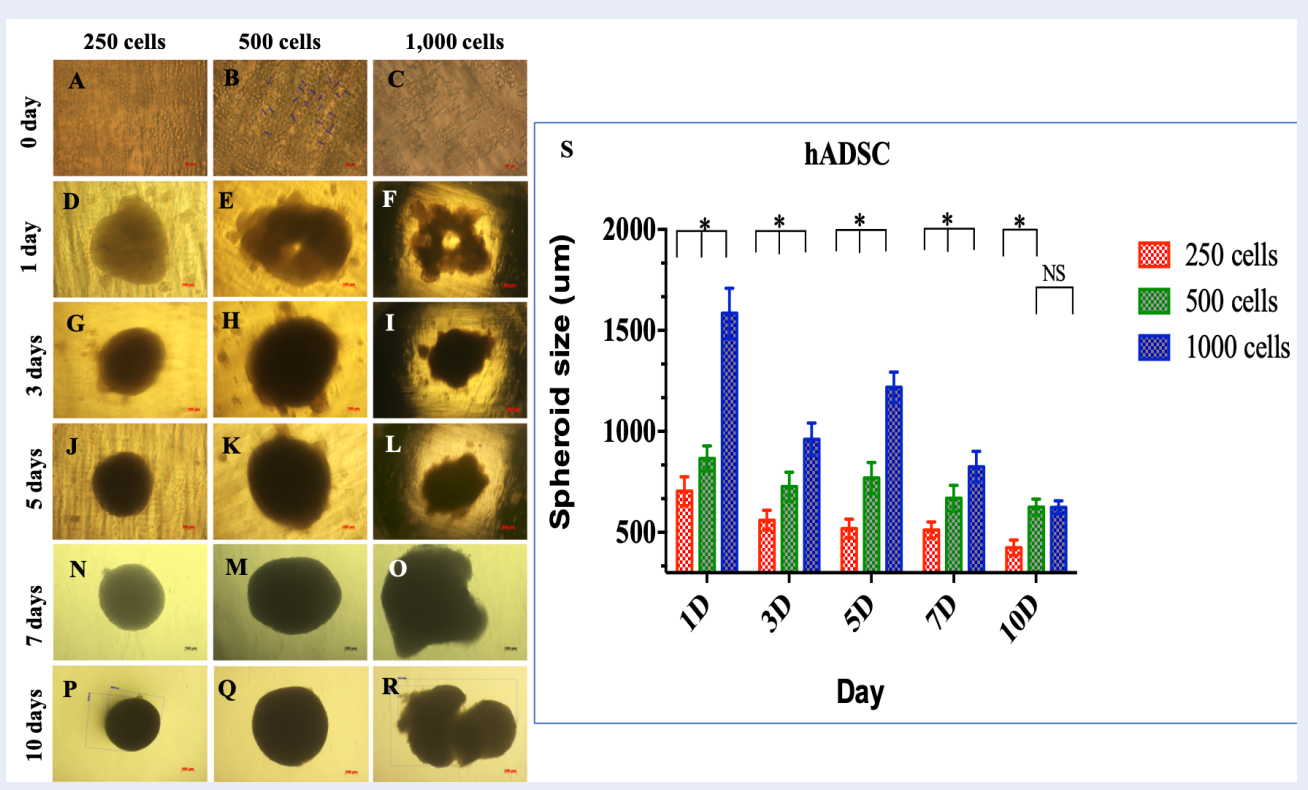

Figure 3: ADSC spheroids in culture. Single cells were seeded in the plates at a density of 250 cells/well. (A), 500 cells/well. (B) 1,000 cells/well. (C). Cell clusters were formed at day 1 (D-F), at day $3(\mathbf{G}, \mathbf{H})$, day $5(\mathbf{J}, \mathbf{K})$, day 7 $(\mathbf{N}, \mathbf{M})$, and day $10(\mathbf{P}, \mathbf{Q})$, in the density group of 250 cells/well $(\mathbf{D}, \mathbf{G}, \mathbf{J}, \mathbf{N}, \mathbf{P})$ and 500 cells/well $(\mathbf{H}, \mathbf{J}, \mathbf{M}, \mathbf{Q})$, but not in the density group of 1,000 cells/well $(\mathbf{F}, \mathbf{I}, \mathbf{L}, \mathbf{O}, \mathbf{R})$. The spheroid size at 250 cells/well and 500 cells/well densities seemed to decrease over time, while at the 1,000 cells/well density, there was no set pattern (S). $\left.*^{*}: p<0.05\right)$.

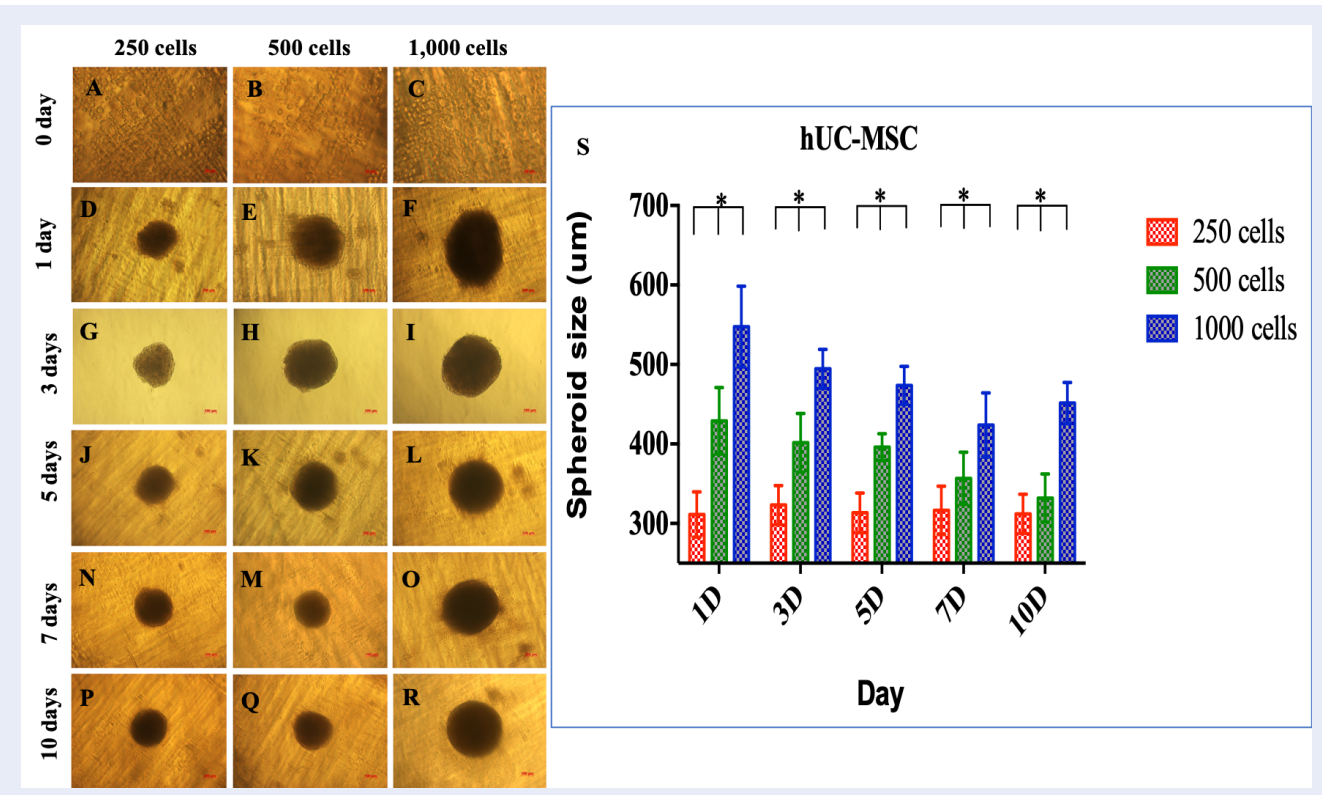

Figure 4: UCMSC spheroids in culture. Single cells were seeded in plate at a density of 250 cells/well (A), 500 cells/well (B), or 1,000 cells/well (C). Spheroids were formed at day 1 (D-F), day $3(\mathbf{G}-\mathbf{I})$, day 5 (J-L), day 7 (N-O), and day 10 (P-R) in the seeding density group of 250 cells/well (D, G, J, N, P), 500 cells/well (H, J, M, Q), and 1,000 cells/well $(\mathbf{F}, \mathbf{I}, \mathbf{L}, \mathbf{O}, \mathbf{R})$. The spheroid size at a density of 250 cells/well, 500 cells/well, and 1,000 cells/well were decreased over time $(\mathbf{S}) .\left(^{*}: \mathbf{p}<0.05\right)$. 
Table 2: UCMSC spheroids formed at different cell densities

\begin{tabular}{ccccccc}
\hline $\begin{array}{c}\text { Cell seed } \\
\text { density }\end{array}$ & $\begin{array}{c}\text { Spheroid size at } 250 \text { cells } \\
(\mu \mathbf{m})\end{array}$ & \multicolumn{2}{c}{$\begin{array}{c}\text { Spheroid size at 500 cells } \\
(\mu \mathbf{m})\end{array}$} & $\begin{array}{c}\text { Spheroid size at 1,000 cells } \\
(\mu \mathbf{m})\end{array}$ \\
\hline Time (Day) & Mean & SD & Mean & SD & Mean & SD \\
1 & 311.30 & 28.39 & 429.24 & 41.71 & 547.66 & 50.72 \\
3 & 323.20 & 24.49 & 401.59 & 36.91 & 494.61 & 24.37 \\
5 & 313.45 & 24.96 & 396.07 & 16.97 & 473.83 & 23.86 \\
7 & 316.70 & 30.38 & 356.83 & 32.79 & 423.88 & 40.33 \\
10 & 312.20 & 24.55 & 332.00 & 30.16 & 451.48 & 25.96 \\
\hline
\end{tabular}

density of 1,000 cells/well, many small spheroids clustered to form a larger spheroid (Figure 5E). Therefore, we did not observe homogeneity in the shape or size of this group. On day 10, the distribution of cells inside the spheroids obtained from seeding densities of 250 and 500 cells/well were markedly denser (Figure 5B and D, respectively). However, at high density ( 1,000 cells/well), the presence of cells inside the spheroid core was not observed (Figure 5F). These results demonstrate that ADSCs were able to proliferate inside the spheroid only at lower seeding density. However, at the higher density (1,000 cells/well), cell survival inside the spheroid core was weak because there were no observed cells at the spheroid core. These findings suggest that a seeding density of 1,000 cells/well is not suitable for ADSCs to create spheroids.

For UCMSC spheroids, the cell distribution for all three density groups $(250,500$, and 1,000 cells/well) at day 3 was uniform (Figure 6A, C and E, respectively). The cells were evenly distributed from inside to outside of the spheroids. Furthermore, the shape and size of the spheroids were homogeneous. The cell distribution was maintained until day 10 . Indeed, on day 10 , the cell compression was highest for all three density groups (250, 500, and 1,000 cells/well (Figure 6B, $\mathbf{D}$, and $\mathbf{F}$, respectively). These results demonstrate that UCMSCs were capable of proliferating inside the spheroids and that the cell distribution was homogeneous, spheroid shape, and size for all three density groups.

\section{Cell survival in MSC spheroids}

No dead cells were observed until day 10 in the ADSC spheroids at the 250 cells/well seeding density (Figure 7A-D). Indeed, 100\% of the cell nuclei were stained blue with Hoechst 33342, and are shown in Figure 7A). Meanwhile, on day 10, red-colored nuclei (stained with PI) were observed in the 500 cells/well density group (Figure 7F) and 1,000 cells/well group
(Figure $7 \mathbf{K}$ ). Since the blue color represents viable cells and the red color represents dead cells, our results show that there is a high presence of dead cells in ADSC spheres- notably for the 500 cells/well seeding group but especially for the 1000 cells/well seeding group- in addition to the presence of live cells (Figure 7E-M).

For the UCMSC group, there was no presence of dead cells inside the spheroids at the 250 cells/well seeding density (Figure 8A-D). However, there were a small number of nuclei inside the spheroid center, which stained positive with PI (Figure 8F) and thus represented dead cells at the seeding density of 500 cells/well (Figure 8E-H). The percentage of dead cells was higher for the 1,000 cells/well density group (Figure 8I-M) at day 10; these results are consistent with those for ADSC spheroids.

\section{Spheroid histology}

Spheroid sections were stained with H\&E, revealing the distribution of mass inside the spheroids (Figure 9). H\&E staining results demonstrated that on day 10, the layer of cells was arranged close together and appeared densely packed at the density of 250 cells/well- in both human ADSC (Figure 9D) and UCMSC spheroids (Figure 9A). Moreover, the spheroids generally became less spherical as spheroid diameter increased (Figure 9C, F). On the other hand, the necrotic core presence was observed at the density of 500 (Figure 9B, E) and 1000 cells/well (Figure 9C, F) in human UCMSC (Figure 9B, C) and human ADSC (Figure 9E, F) spheroid at day 10.

\section{DISCUSSION}

In the present study, we successfully produced ADSCand UCMSC-derived spheroids and compared the efficiency of creating spheroids from these MSC sources. In the first assay, the results showed that ADSCs and UCMSCs exhibited the three minimal defining criteria for MSCs, according to the proposed 
3D structure -3 days
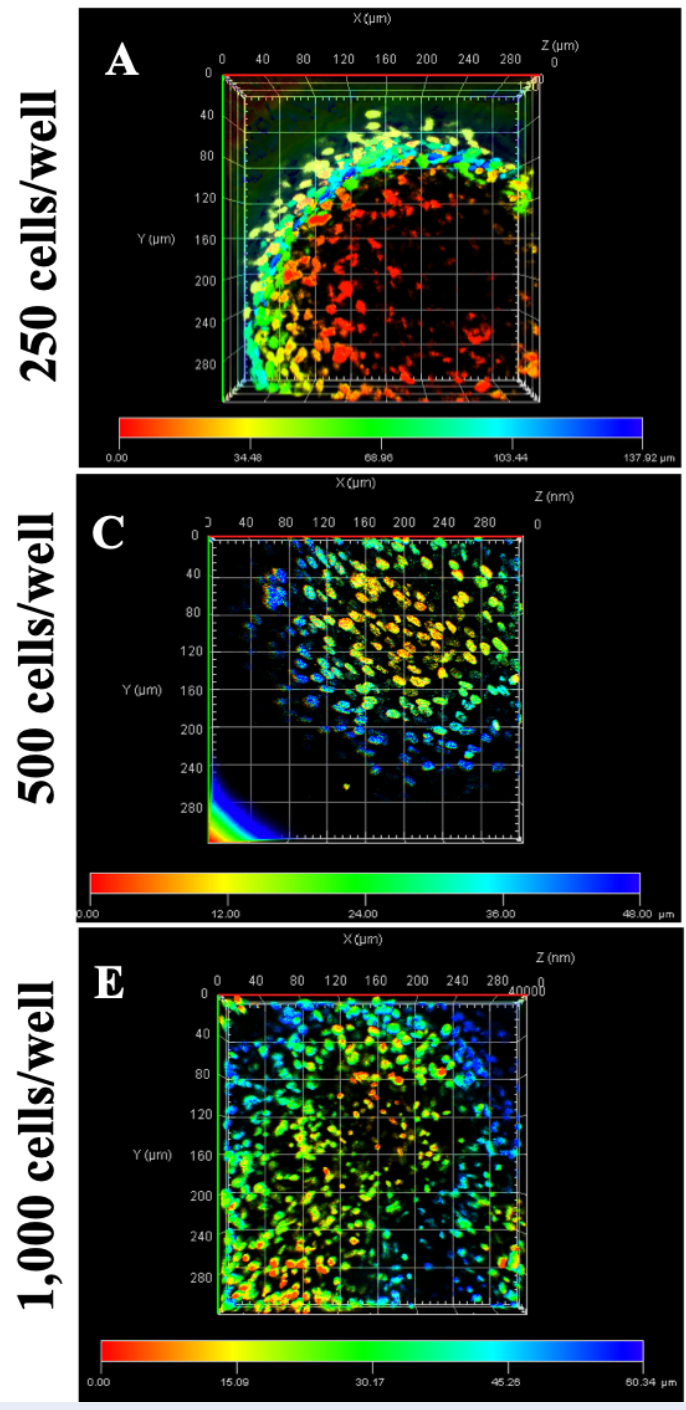

Figure 5: Cell compression in ADSC spheroids. Compression of ADSC at day 3 for seeding densities of 250 (A), $500(\mathbf{C})$, and 1,000 cells/well (E). ADSC cell numbers increased until day 10 (B, D, F). The loss of cells was observed at a seeding density of 1,000 cells/well on day 10 (F) (red to blue: inner core to outer spheroid).

guidelines of the International Society for Cellular Therapy $^{27}$. First, ADSCs and UCMSCs could adhere to a plastic surface and displayed the classic fibroblast-like morphology. Second, both cell lines were positive for the expression of surface markers CD90, CD44, CD73, and CD105, but were negative for CD14, CD34, CD45, and HLA-DR. Finally, both cell lines had the ability to differentiate into other functional cells, such as adipocytes, osteocytes, and chondrocytes. These results are in accordance with published studies by Choudhery, et al. ${ }^{9}$.

\section{D structure -10 days}
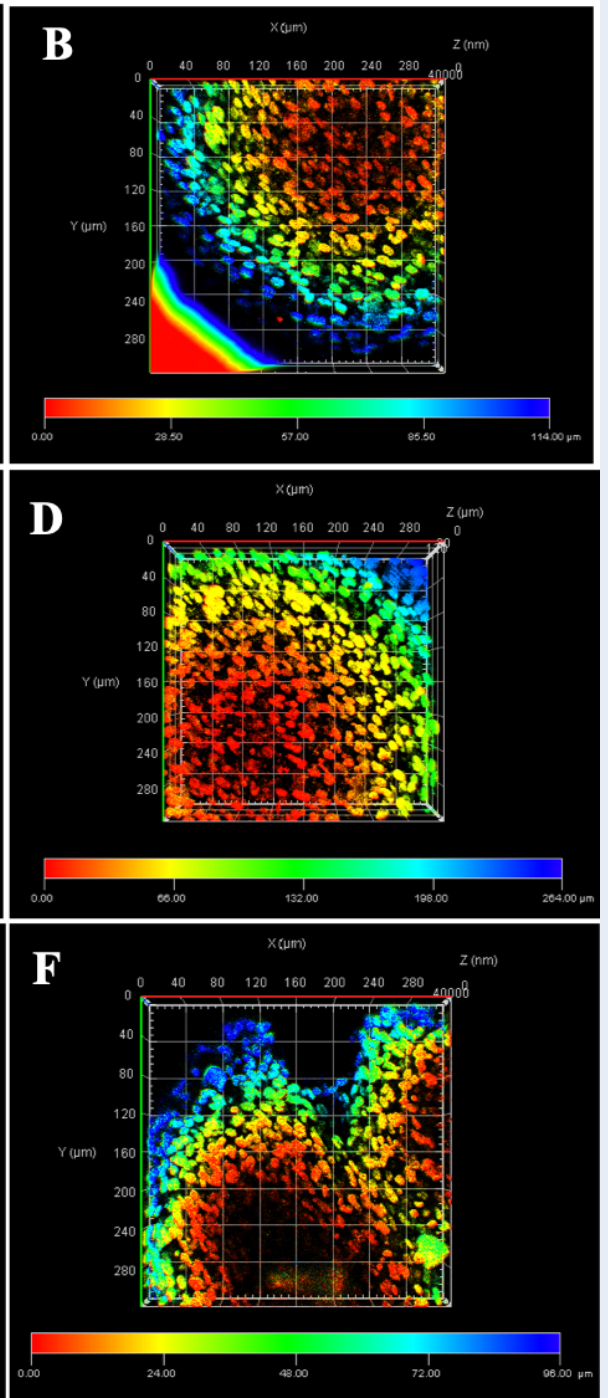


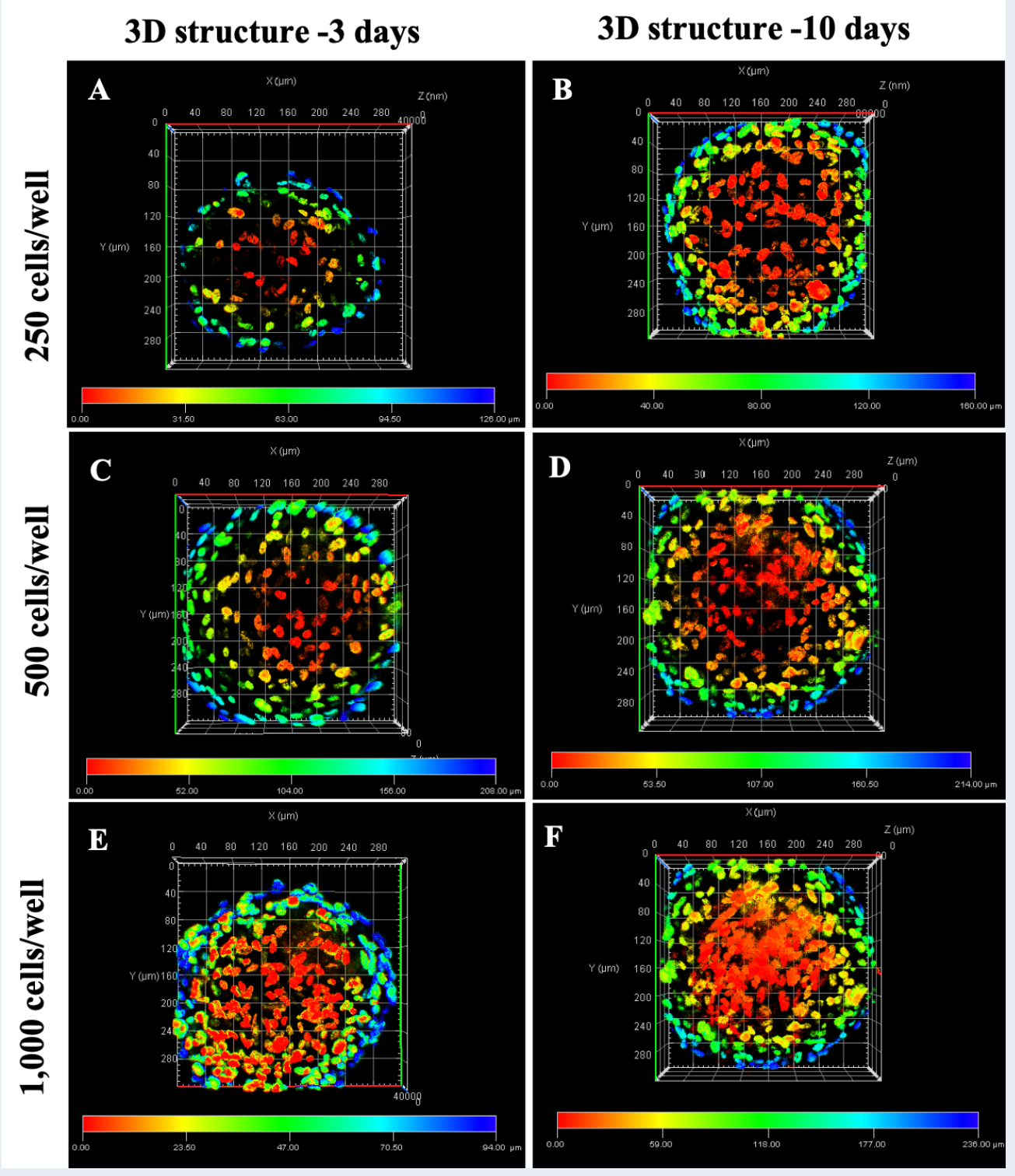

Figure 6: Cell compression in human UCMSC spheroids. Compression of UCMSC spheroids at day 3 after seeding density of 250 (A), 500 (C), and 1,000 cells/well (E). The cell numbers increased until day 10 (B, D, F). The cell distribution, spheroid shape, and size were remarkably homogeneous until day 10 (A-F) (red to blue: inner to outer spheroid).

ADSC spheroids at cell seeding densities below 1,000 cells/well.

During the ten days of culture, the spheroid size tended to decrease. However, the cell density of the spheroids tended to increase. This may be due to cell compression in the spheroid. The compactness of spheroids is thought to be based on the homophilic cadherin-cadherin binding at the membrane surface $^{28}$. E-cadherin expression is low in monolayer cell culture and high in 3D cell culture. The high expres- sion of E-cadherin produced in cell clusters can affect the spheroid compaction ${ }^{29}$.

On the other hand, the spheroids' volume was compressed to a certain extent, which inhibited the inner cell layer in the core from accessing nutrients and oxygen, while the $\mathrm{CO}_{2}$ concentration increased. Theoretically, this could lead to slower growth over time, and the spheroids could eventually stop growing in size by day 10. According to the research on MSC spheroids by Kaitlin C. Murphy et al. ${ }^{30}$, cells' via- 


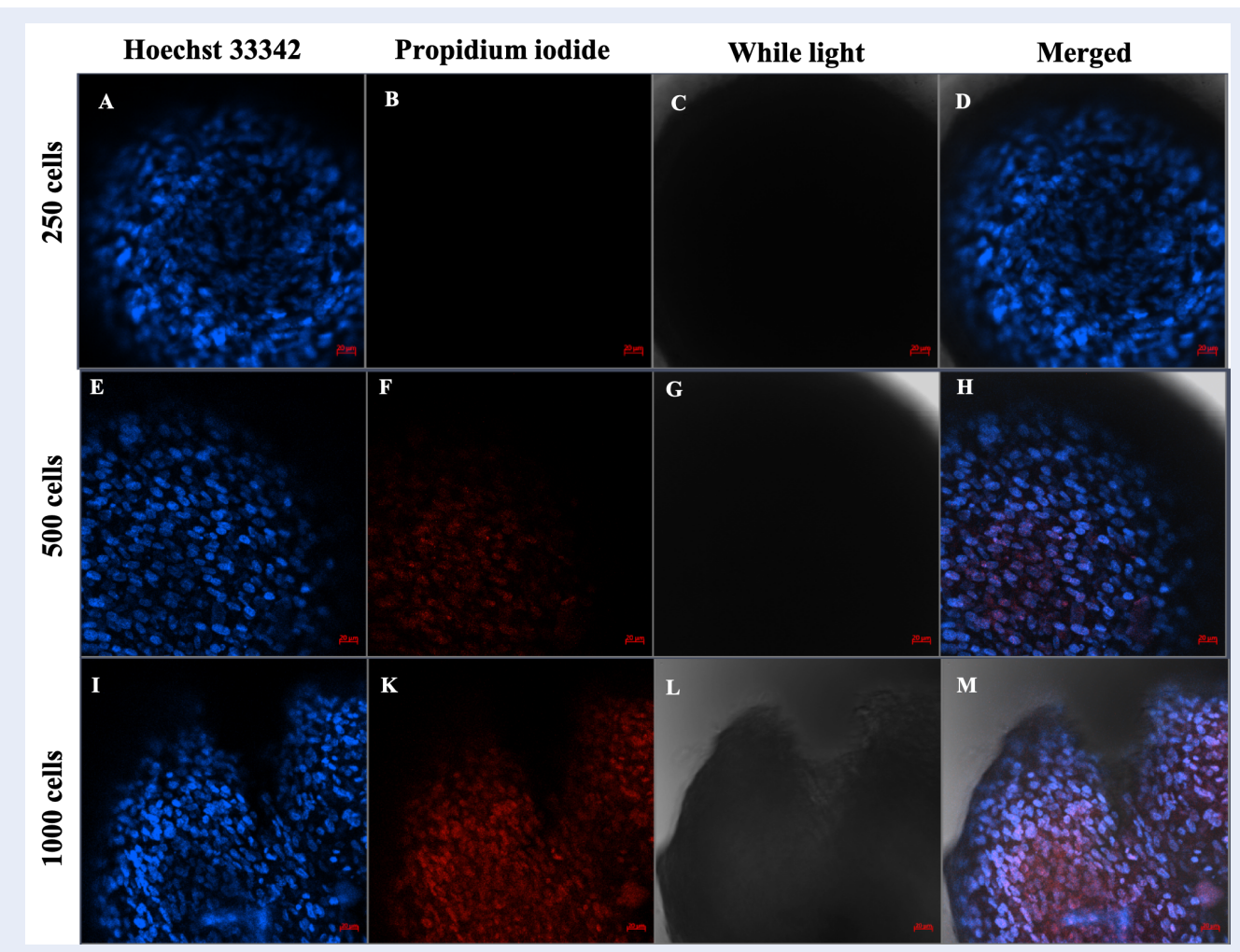

Figure 7: Cell death and survival in ADSC spheroids at day 10. Dead cells stained with propidium nuclei (redcolored nuclei); live cells stained with Hoechst 33342 (blue-colored nuclei).

bility and metabolic activity decreased with increasing spheroid size (e.g., at the seeding density of 1,000 cells/well for human ADSC spheroids). Our study herein showed similar results for the seeding density of 500 and 1,000 cells/well.

Notably, at high density (1,000 cells/well), ADSC spheroid shape was not defined. This may be due to the fact that the cells were seeded in a limited environment. Thus, the outer layer can easily flake off, making it challenging for the spheroids to maintain their shape for a long period. The above analysis provides important insight into the consideration of suitable seeding densities to create the desired spheroids in the shortest period, with the maximal number of living cells and using the lowest amount of initial cells. Spheroids were also evaluated for the ratio of live to dead cells via propidium iodide (PI)/Hoechst 33342 double staining. PI is an impermeable membrane dye that can identify dead cells with a red fluorescence ${ }^{31}$, while the blue fluorescent Hoechst 33342 is a cellpermeable nucleic acid that is widely used to stain live cells ${ }^{32}$. Our results showed that at the density of 250 cells/well, the survival of ADSCs and UCMSCs in spheroids is the same until day 10. At this time point (day 10), it is best to compare the two other densities (500 and 1,000 cells/well). Dead cells' presence was not observed if the initial cell seeding density was less than 250 cells/well or if the spheroid culture time was shorter than three days. However, at a density of 500 cells/well for both ADSC and UCMSC spheroid and a density of 1,000 cells/well for UCMSC spheroid, a complete spheroid was formed with a structure that included the three zones (proliferating, quiescent, and necrotic zones).

Moreover, a stable morphology was maintained until day 10. At the densities of 500 and 1,000 cells/well for the same cell culture time, the presence of viable cells in UCMSC spheroids was higher than that for ADSC spheroids. The dead cell were formed inside the center of the spheroid and dependent on initial cell density, time to culture, and spheroid diameter. The necrosis core formation is caused by the lack of nutrients and $\mathrm{O}_{2}$ in the core's inner cell layer, while $\mathrm{CO}_{2}$ concentration was increased in the core ${ }^{33,34}$. The dead cells inside and surrounding the necrotic core can secrete substances containing enzymes that may cause the spheroids' decomposition.

Moreover, cell-cell contact in 3D cell culture is another predominant reason for necrosis ${ }^{35}$. At the den- 


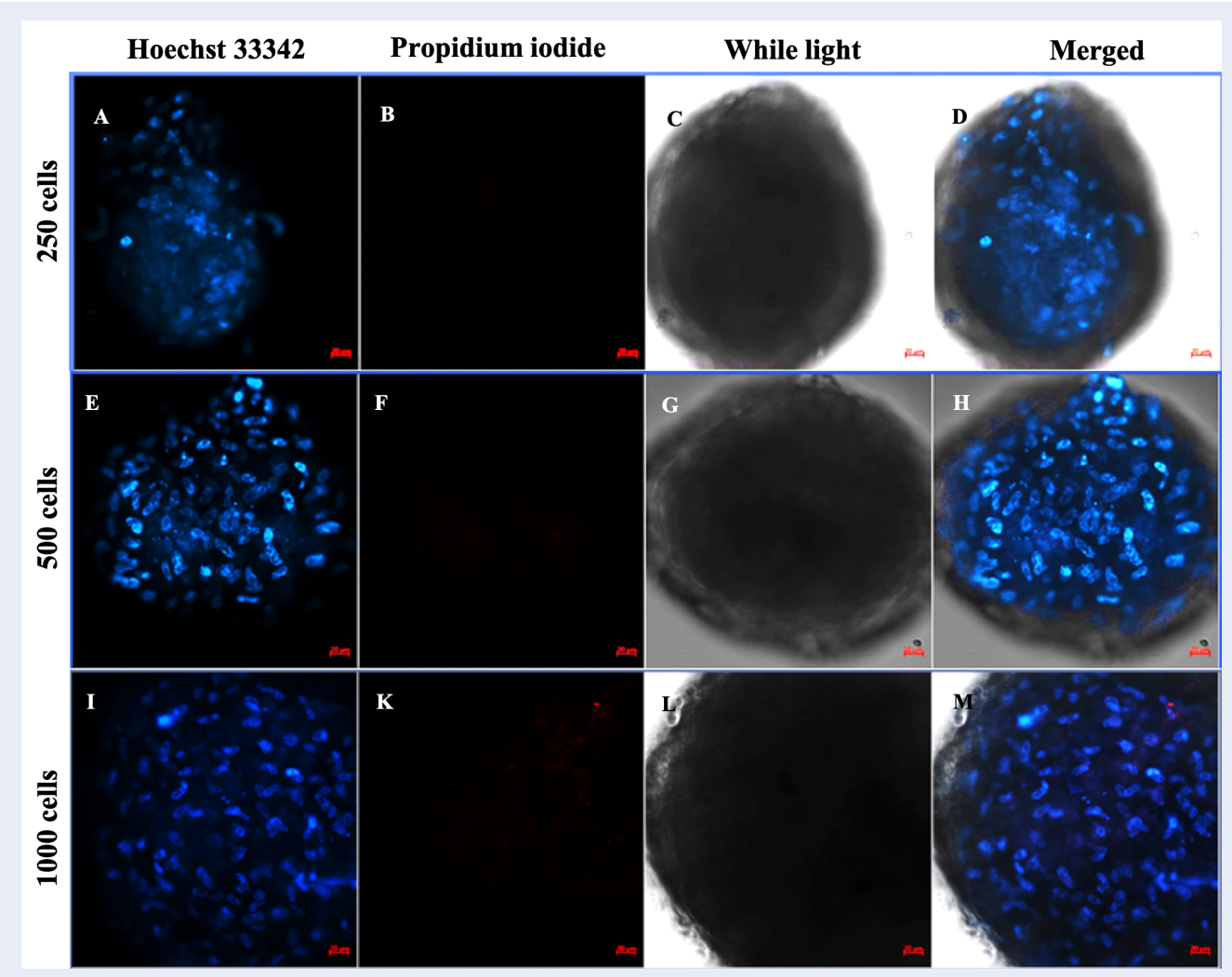

Figure 8: Cell death and survival of UCM SC spheroids at day 10. Dead cells stained with propidium nuclei (red-colored nuclei); live cells stained with Hoechst 33342 (blue-colored nuclei).

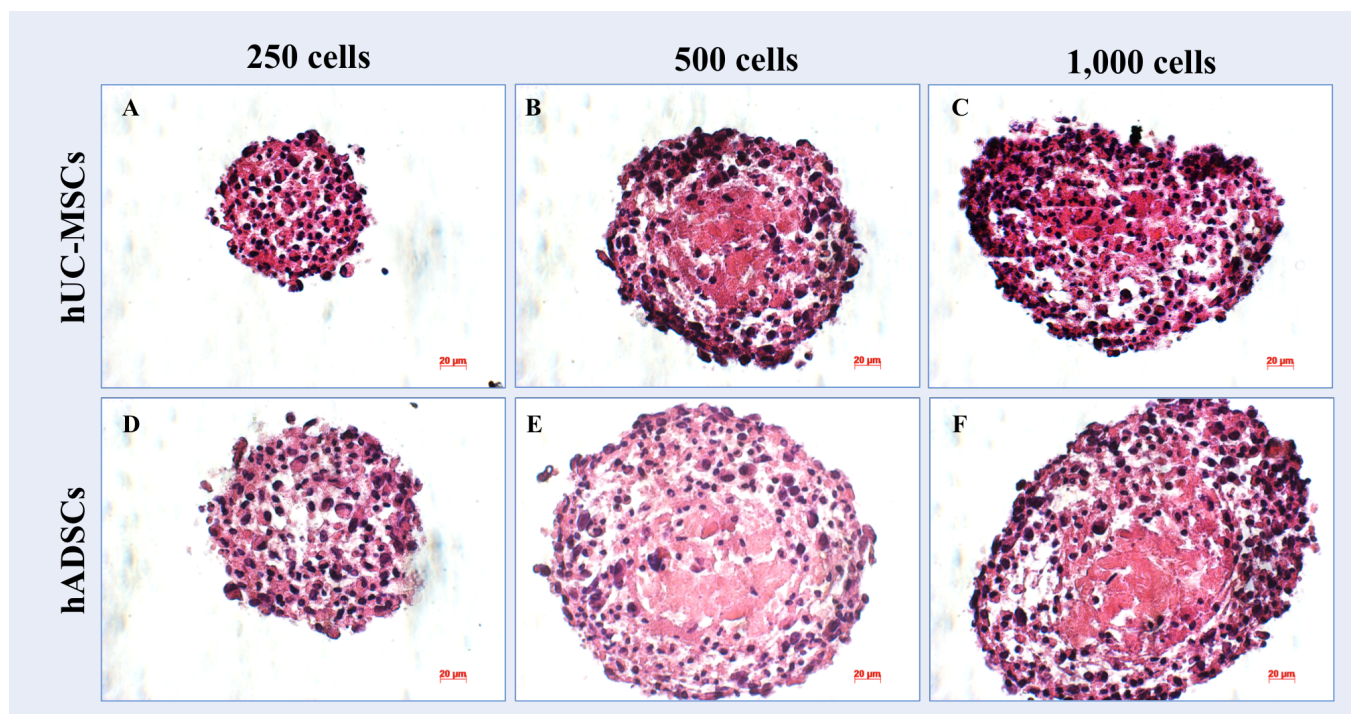

Figure 9: Spheroid histology. The layer of cells was arranged close together and appeared densely packed at a density of 250 cells/well in both ADSC (D) and UCMSC spheroids (A). The necrotic core presence was observed at the density of $500(\mathbf{B}, \mathbf{E})$ and 1000 cells/well $(\mathbf{C}, \mathbf{F})$ in $\operatorname{UCMSC}(\mathbf{B}, \mathbf{C})$ and $\operatorname{ADSC}(\mathbf{E}, \mathbf{F})$ spheroid on day 10. 
sity of 500 cells/well, for the ADSC spheroids, while there were some dead cells in the hypoxic core, the spheroids still maintained the ability to proliferate during the 10-day period. Furthermore, there was an increase in the number of cells and the size was maintained (compared to the results of the other seeding density groups); these factors can shorten the cultivation time of the spheroids and enable spheroids to form while avoiding the creation of the necrosis core.

\section{CONCLUSION}

In the present study, we successfully produced spheroids from ADSCs and UCMSCs and compared the efficiency of spheroid formation from these sources of MSCs. Overall, the results suggest that UCMSC are more effective in spheroid formation than UCMSC at each of the investigated cell densities. A necrotic core inside the spheroid was formed depending on the initial cell density, expansion time, and diameter of spheroids. Dead cells were not present in the spheroids when the initial cell seeding density was less than 250 cells/well, or when expansion time was shorter than three days. When seeding at the same density, the size of spheroids derived from ADSCs was found to be larger than that from UCMSC. Taken together, these results suggest that spheroids can be produced from ADSCs and UCMSCs, at a cell density of 250 cells/well, in non-adherent V-bottom 96-well plates. This process can be scaledup using liquid handling robots. With the method described above, a large number of spheroids from $\mathrm{AD}$ SCs or UCMSCs can be generated and applied in tissue engineering as engineered cartilage or bone tissues.

\section{ABBREVIATIONS}

2D: Two-dimensional culture

3D: Three-dimensional culture

ADSCs: Human Adipose-Derived Stem Cells

ECM: Extracellular matrix

EGF: Epidermal Growth Factor

FBS: Fetal Bovine Serum

GFG: Fibroblast Growth Factor GAGs Glycosaminoglycans

HLA-DR: Human Leukocyte Antigen - antigen D Related

IBMX: 1 - methyl - 3 - isobutylxanthine

UCMSCs: Human Umbilical Cord Mesenchymal Stem Cells

SD: Standard Deviation

\section{ACKNOWLEDGEMENTS}

This research is approved by the Vietnam National University Ho Chi Minh City (VNUHCM). Authors thank Van Hanh General Hospital, Ho Chi Minh City, Viet Nam approved to use the adipose tissues from donors for the study. We thank General Hospital District 2, Ho Chi Minh City, Viet Nam approved for using the umbilical cord tissues from donors for this study.

\section{AUTHOR'S CONTRIBUTIONS}

All authors equally contributed to this study. All authors read and approved the final manuscript.

\section{FUNDING}

This research is funded by Vietnam National University Ho Chi Minh City (VNU-HCM) under grant number TX2018-18-02.

\section{AVAILABILITY OF DATA AND MATERIALS}

Data and materials used and analyzed during the current study are available from the corresponding author on reasonable request.

\section{ETHICS APPROVAL AND CONSENT TO PARTICIPATE}

The institutional review board approved the use of human tissues for study.

\section{CONSENT FOR PUBLICATION}

Not applicable.

\section{COMPETING INTERESTS}

The authors declare that they have no competing interests.

\section{REFERENCES}

1. Edmondson R, et al. Three-dimensional cell culture systems and their applications in drug discovery and cellbased biosensors. Assay and drug development technologies. 2014;12(4):207-218. PMID: 24831787. Available from: https: //doi.org/10.1089/adt.2014.573.

2. Baraniak PR, McDevitt TC. Scaffold-free culture of mesenchymal stem cell spheroids in suspension preserves multilineage potential. Cell Tissue Res. 2012;347(3):701-711. PMID: 21833761. Available from: https://doi.org/10.1007/s00441011-1215-5.

3. Antoni $\mathrm{D}$, et al. Three-dimensional cell culture: a breakthrough in vivo. International journal of molecular sciences. 2015;16(3):5517-5527. PMID: 25768338. Available from: https://doi.org/10.3390/ijms16035517.

4. Anderer $\mathrm{U}$, Libera J. In vitro engineering of human autogenous cartilage. Journal of bone and mineral research. 2002;17(8):1420-1429. PMID: 12162496. Available from: https://doi.org/10.1359/jbmr.2002.17.8.1420.

5. Kuo CT, et al. Three-dimensional spheroid culture targeting versatile tissue bioassays using a PDMS-based hanging drop array. Scientific Reports. 2017;7(1):4363. PMID: 28663555. Available from: https://doi.org/10.1038/s41598-017-04718-1. 
6. Ivascu A, Kubbies M. Diversity of cell-mediated adhesions in breast cancer spheroids. Int J Oncol. 2007;31(6):1403-1413. PMID: 17982667. Available from: https://doi.org/10.3892/ijo. 31.6.1403.

7. Han C, Takayama S, Park J. Formation and manipulation of cell spheroids using a density adjusted PEG/DEX aqueous two phase system. Scientific Reports. 2015;5:11891. PMID: 26144552. Available from: https://doi.org/10.1038/srep11891.

8. Huselstein C, Li Y, He X. Mesenchymal stem cells for cartilage engineering. Bio-medical materials and engineering. 2012;22(1-3):69-80. PMID: 22766704. Available from: https: //doi.org/10.3233/BME-2012-0691.

9. Choudhery MS. Comparison of human mesenchymal stem cells derived from adipose and cord tissue. Cytotherapy. 2013;15(3):330-343. PMID: 23318344. Available from: https: //doi.org/10.1016/j.jcyt.2012.11.010.

10. Wang HS, et al. Mesenchymal stem cells in the Wharton's jelly of the human umbilical cord. Stem cells. 2004;22(7):13301337. PMID: 15579650. Available from: https://doi.org/10. 1634/stemcells.2004-0013.

11. Yoon $\mathrm{HH}$, et al. Enhanced cartilage formation via threedimensional cell engineering of human adipose-derived stem cells. Tissue Engineering Part A. 2012;18(19-20):1949-1956. PMID: 22881427. Available from: https://doi.org/10.1089/ten. tea.2011.0647.

12. Zuk PA, et al. Human adipose tissue is a source of multipotent stem cells. Molecular biology of the cell. 2002;13(12):42794295. PMID: 12475952. Available from: https://doi.org/10. 1091/mbc.e02-02-0105.

13. Winter A, et al. Cartilage-like gene expression in differentiated human stem cell spheroids: a comparison of bone marrowderived and adipose tissue-derived stromal cells. Arthritis Rheum. 2003;48(2):418-429. PMID: 12571852. Available from: https://doi.org/10.1002/art.10767.

14. Zhang $\mathrm{K}$, et al. In-situ birth of MSCs multicellular spheroids in poly(L-glutamic acid)/chitosan scaffold for hyaline-like cartilage regeneration. Biomaterials. 2015;71:24-34. PMID: 26318814. Available from: https://doi.org/10.1016/j.biomaterials.2015.08.037.

15. Zhang $K$, et al. Functionalized Scaffold for in Situ Efficient Gene Transfection of Mesenchymal Stem Cells Spheroids toward Chondrogenesis. ACS Appl Mater Interfaces. 2018;10(40):33993-34004. PMID: 30207161. Available from: https://doi.org/10.1021/acsami.8b12268.

16. Stuart MP, et al. Successful Low-Cost Scaffold-Free Cartilage Tissue Engineering Using Human Cartilage Progenitor Cell Spheroids Formed by Micromolded Nonadhesive Hydrogel. Stem Cells International. 2017;2017:7053465. PMID: 29527227. Available from: https://doi.org/10.1155/2017/ 7053465.

17. Tu VTK, et al. Method for in production of cartilage from scaffold-free spheroids composed of human adipose-derived stem cells. Biomedical Research and Therapy. 2020;7(4):36973708. Available from: https://doi.org/10.15419/bmrat.v7i4.597.

18. Shen $\mathrm{FH}$, et al. Implications of adipose-derived stromal cells in a 3D culture system for osteogenic differentiation: an in vitro and in vivo investigation. Spine J. 2013;13(1):32-43. PMID: 23384881. Available from: https://doi.org/10.1016/j.spinee. 2013.01.002.

19. Laschke MW, et al. In vitro osteogenic differentiation of adipose-derived mesenchymal stem cell spheroids impairs their in vivo vascularization capacity inside implanted porous polyurethane scaffolds. Acta Biomater. 2014;10(10):42264235. PMID: 24998773. Available from: https://doi.org/10. 1016/j.actbio.2014.06.035.

20. Fennema EM, et al. Ectopic bone formation by aggregated mesenchymal stem cells from bone marrow and adipose tissue: A comparative study. J Tissue Eng Regen Med. 2018;12(1):e150-e158. PMID: 28485099. Available from: https://doi.org/10.1002/term.2453.

21. Murata $D$, et al. A preliminary study of osteochondral regeneration using a scaffold-free three-dimensional construct of porcine adipose tissue-derived mesenchymal stem cells. J Orthop Surg Res. 2015;10:35. PMID: 25890366. Available from: https://doi.org/10.1186/s13018-015-0173-0.

22. Ahmad T, et al. Fabrication of in vitro $3 \mathrm{D}$ mineralized tissue by fusion of composite spheroids incorporating biomineralcoated nanofibers and human adipose-derived stem cells. Acta Biomater. 2018;74:464-477. PMID: 29803004. Available from: https://doi.org/10.1016/j.actbio.2018.05.035.

23. Pham PV, et al. Isolation and proliferation of umbilical cord tissue derived mesenchymal stem cells for clinical applications. Cell Tissue Bank. 2016;17(2):289-302. PMID: 26679929. Available from: https://doi.org/10.1007/s10561-015-9541-6.

24. Cui $X$, Hartanto $Y$, Zhang $H$. Advances in multicellular spheroids formation. Journal of the Royal Society Interface. 2017;14(127):20160877. PMID: 28202590. Available from: 10.1098/rsif.2016.0877.

25. Truong NC, Bui KH, Pham PV. Characterization of Senescence of Human Adipose-Derived Stem Cells After Long-Term Expansion. Adv Exp Med Biol. 2019;1084:109-128. PMID: 30242785. Available from: https://doi.org/10.1007/5584_ 2018_235.

26. Pham PV, et al. Good manufacturing practice-compliant isolation and culture of human adipose-derived stem cells. Biomedical Research and Therapy. 2014;1(04):133-141. Available from: https://doi.org/10.7603/s40730-014-0021-6.

27. Dominici $M$, et al. Minimal criteria for defining multipotent mesenchymal stromal cells. The International Society for Cellular Therapy position statement. Cytotherapy. 2006;8(4):315-317. PMID: 16923606. Available from: https: //doi.org/10.1080/14653240600855905.

28. Cui $X$, Hartanto $Y$, Zhang $H$. Advances in multicellular spheroids formation. Journal of The Royal Society Interface. 2017;14(127):20160877. PMID: 28202590. Available from: https://doi.org/10.1098/rsif.2016.0877.

29. Schmidt M, et al. Spheroid-based 3-dimensional culture models: Gene expression and functionality in head and neck cancer. Oncol Rep. 2016;35(4):2431-2440. PMID: 26797047. Available from: https://doi.org/10.3892/or.2016.4581.

30. Murphy KC, et al. Measurement of oxygen tension within mesenchymal stem cell spheroids. Journal of The Royal Society Interface. 2017;14(127):20160851. PMID: 28179546. Available from: https://doi.org/10.1098/rsif.2016.0851.

31. Boyd V, Cholewa OM, Papas KK. Limitations in the Use of Fluorescein Diacetate/Propidium lodide (FDA/PI) and Cell Permeable Nucleic Acid Stains for Viability Measurements of Isolated Islets of Langerhans. Current trends in biotechnology and pharmacy. 2008;2(2):66-84.

32. Rahman SA, et al. In Vitro Morphological Assessment of Apoptosis Induced by Antiproliferative Constituents from the Rhizomes of Curcuma zedoaria. Evidence-Based Complementary and Alternative Medicine. 2013;2013:14. PMID: 23762112. Available from: https://doi.org/10.1155/2013/257108.

33. Xu J, Vilanova G, Gomez H. A Mathematical Model Coupling Tumor Growth and Angiogenesis. PLoS One. 2016;11(2):e0149422. PMID: 26891163. Available from: https://doi.org/10.1371/journal.pone.0149422.

34. Barisam M, et al. Prediction of Necrotic Core and Hypoxic Zone of Multicellular Spheroids in a Microbioreactor with a U-Shaped Barrier. Micromachines. 2018;9(3):94. PMID: 30424028. Available from: https://doi.org/10.3390/mi9030094.

35. Bizik J, et al. Cell-cell contacts trigger programmed necrosis and induce cyclooxygenase-2 expression. Cell Death Differ. 2004;11(2):183-195. PMID: 14555963. Available from: https://doi.org/10.1038/sj.cdd.4401317. 